\title{
Linear and nonlinear rheology of wormlike micelles
}

\author{
A K SOOD, RANJINI BANDYOPADHYAY and GEETHA BASAPPA \\ Department of Physics, Indian Institute of Science, Bangalore 560012 , India
}

\begin{abstract}
Several surfactant molecules self-assemble in solution to form long, cylindrical, flexible wormlike micelles. These micelles can be entangled with each other leading to viscoelastic phases. The rheological properties of such phases are very interesting and have been the subject of a large number of experimental and theoretical studies in recent years. We shall report our recent work on the macrorheology, microrheology and nonlinear flow behaviour of dilute aqueous solutions of a surfactant CTAT (Cetyltrimethylammonium Tosilate). This system forms elongated micelles and exhibits strong viscoelasticity at low concentrations $(\sim 0.9 \mathrm{wt} \%)$ without the addition of electrolytes. Microrheology measurements of $G(\omega)$ have been done using diffusing wave spectroscopy which will be compared with the conventional frequency sweep measurements done using a cone and plate rheometer. The second part of the paper deals with the nonlinear rheology where the measured shear stress $\sigma$ is a nonmonotonic function of the shear rate $\dot{\gamma}$. In stresscontrolled experiments, the shear stress shows a plateau for $\dot{\gamma}$ larger than some critical strain rate, similar to the earlier reports on $\mathrm{CPyCl} / \mathrm{NaSal}$ system. Cates et al have proposed that the plateau is a signature of mechanical instability in the form of shear bands. We have carried out extensive experiments under controlled strain rate conditions, to study the time-dependence of shear stress. The measured time series of shear stress has been analysed in terms of correlation integral and Lyapunov exponent to show unambiguously that the behaviour is typical of low dimensional dynamical systems.
\end{abstract}

Keywords. Rheology; wormlike micelles.

PACS Nos 83.50.Fc; 83.50.Gd; 05.45.-a

\section{Introduction}

Rheology is the study of the deformation and flow of matter. Solids and fluids exhibit different flow behaviours under shear. Solids store mechanical energy and are elastic, whereas fluids dissipate energy and are viscous. Complex fluids (e.g. colloids, polymers), owing to their larger length scales which result in low mechanical susceptibilities ( $\sim 10^{2} \mathrm{~Pa}$ as compared to $\sim 10^{12} \mathrm{~Pa}$ in atomic systems), show very complex flow behaviour and are viscoelastic. The relative proportion of elastic and viscous responses depends on the frequency of the applied stress. For example, for entangled polymer solutions, the stress relaxation predominantly occurs by reptation dynamics with time scales $\tau_{\text {rep }}$. This system will be more elastic for $\omega>\tau_{\text {rep }}^{-1}$ whereas it will be more viscous for $\omega<\tau_{\text {rep }}^{-1}$. One should also note that for soft condensed matter, the elastic modulus under shear stress is much smaller than the elastic modulus under compressive stress whereas these two moduli are nearly equal for conventional atomic systems.

Experiments on the rheology of matter involve the measurement and prediction of its flow behaviour. The method involves the application of a known strain or strain rate to a 


\section{A K Sood, Ranjini Bandyopadhyay and Geetha Basappa}

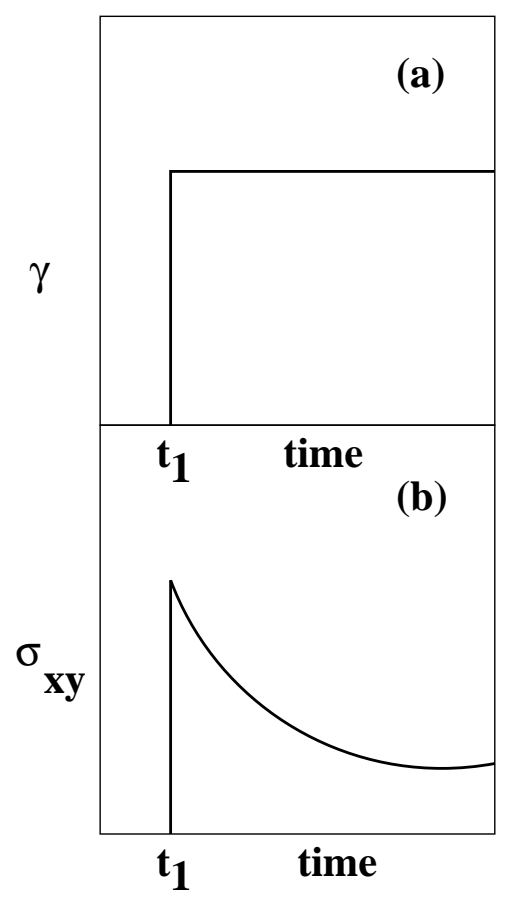

Figure 1. (a) shows step strain applied at time $t_{1}$, with a magnitude small enough to lie in the linear regime (b) shows the corresponding relaxation of stress.

sample and the subsequent measurement of the stress induced in the sample or vice versa. The response of a viscoelastic material to an applied stress may be characterized as linear or nonlinear depending on the magnitude of the applied stress/strain rate.

\subsection{Linear rheology}

The response of a material is linear when very small stresses (i.e. small compared to the spontaneous thermal fluctuations in the material) are applied. If a small step strain $\gamma$ is applied to a deformable material at time $t=t_{1}$, the stress induced in the material is given by $\sigma_{x y}(t)=G\left(t, t_{1}\right) \gamma(t)$ (figure 1 ), where $G\left(t, t_{1}\right)$ is the stress relaxation function. Here $x$ and $y$ define the velocity and velocity gradient directions, respectively. To linear order in $\gamma$, all other components of stress like $\sigma_{x x}$ are zero. Invoking time-translational symmetry, $G$ depends on the time difference between $t$ and $t_{1}$ i.e. $G\left(t, t_{1}\right)=G\left(t-t_{1}\right)$. Exceptions to time-translational symmetry are found to occur in glassy systems which show aging behaviour. For an arbitrarily small applied strain rate $\dot{\gamma}(t)$, the stress $\sigma_{x y}(t)$ induced in the material is defined as

$$
\sigma_{x y}(t)=\int_{-\infty}^{t} G\left(t-t^{\prime}\right) \dot{\gamma}\left(t^{\prime}\right) \mathrm{d} t^{\prime},
$$

where $G(t)$ is known as the memory kernel for shear response. 


\section{Rheology of wormlike micelles}

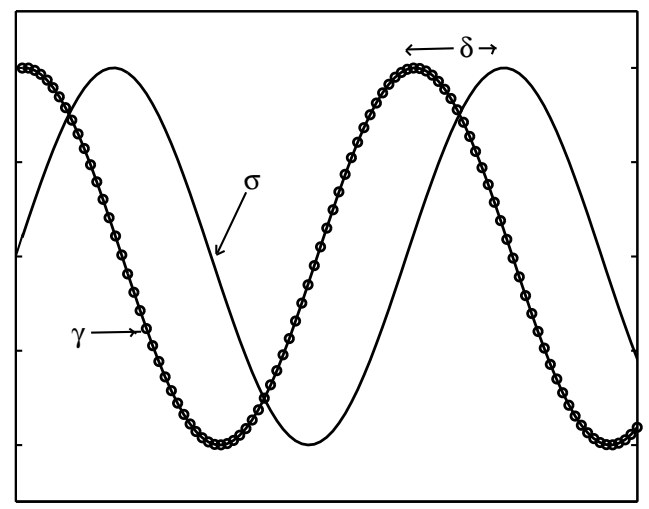

Figure 2. $\gamma$ is the shear applied to a viscoelastic material. $\sigma$ is the resultant stress in the material, delayed by a phase angle $\delta$. The complex shear modulus may be then written as $G^{\star}(\omega)=G^{\prime}(\omega)+i G^{\prime \prime}(\omega)$, where $G^{\prime}(\omega)$ is the in-phase elastic modulus and $G^{\prime \prime}(\omega)$ is the out-of-phase viscous modulus.

(a) Oscillatory flow: If a strain $\gamma(t)=\gamma_{0} \mathrm{e}^{i \omega t}$ is applied to the material, the stress developed in the sample can be out of phase with the strain by a phase angle $\delta$ (figure 2). For a viscoelastic material, $\delta$ lies between the limits $\delta=0$ (for a Hookean solid) and $\delta=\pi / 2$ (for a Newtonian fluid). Using eq. (1), the resulting stress may be written as

$$
\sigma_{x y}(t)=\gamma_{0} \int_{-\infty}^{t} G\left(t-t^{\prime}\right) i \omega \mathrm{e}^{i \omega t^{\prime}} \mathrm{d} t^{\prime} .
$$

Putting $\tau=t-t^{\prime}$, it can be written as $\sigma_{x y}(t)=\gamma_{0} \mathrm{e}^{i \omega t} G^{\star}(\omega), G^{\star}(\omega)=G^{\prime}(\omega)+i G^{\prime \prime}(\omega)$, is the complex shear modulus given by

$$
G^{\star}(\omega)=i \omega \int_{0}^{\infty} G(t) \mathrm{e}^{-i \omega t} \mathrm{~d} t
$$

The real part of $G^{\star}(\omega)$, called the storage modulus and denoted by $G^{\prime}(\omega)$, gives the elastic response of the material to the applied strain. It is the ratio of the stress in phase with the applied strain to the strain. The viscous response, defined by the loss modulus $G^{\prime \prime}(\omega)$, is the ratio of the out-of-phase stress component to the strain. Equation (3) implies that $G^{\prime}(\omega)$ is an even function whereas $G^{\prime \prime}(\omega)$ is an odd function of $\omega$.

(b) Steady shear: When $\dot{\gamma}(t)=$ constant, the stress induced in the sample is given by $\sigma_{x y}=\dot{\gamma} \int_{-\infty}^{t} G\left(t-t^{\prime}\right) \mathrm{d} t^{\prime}=\eta_{0} \dot{\gamma}$, where $\eta_{0}$ is the zero shear viscosity given by $\eta_{0}=$ $\int_{0}^{\infty} G(t) \mathrm{d} t$. A non-zero value of $\eta_{0}$ implies the presence of liquid-like or glassy dynamics.

The simplest form of the response function $G(t)$ is given by the Maxwell model, namely, $G(t)=G_{0} \mathrm{e}^{-t / \tau_{\mathrm{M}}}$, or $G^{\star}(\omega)=G_{0} i \omega \tau_{\mathrm{M}} /\left(1+i \omega \tau_{\mathrm{M}}\right)$, where $G_{0}$ is the elastic modulus and $\tau_{\mathrm{M}}$ is the Maxwell relaxation time related to zero frequency shear viscosity $\eta_{0}=G_{0} \tau_{\mathrm{M}}$. For a Newtonian fluid, $G_{0} \longrightarrow \infty, \tau_{\mathrm{M}} \longrightarrow 0$ such that $\eta_{0}$ remains constant. More generally, when there are many relaxation times present in the system, $G(t)=\sum_{j} G_{j} \mathrm{e}^{-t / \tau_{j}}$. For a continuous distribution of relaxation times, specified in terms of $P(\tau)$,

$$
G(t)=G_{0} \int_{0}^{\infty} P(\tau) \mathrm{e}^{-t / \tau} \mathrm{d} \tau \equiv G_{0} \mu(t)
$$




\section{A K Sood, Ranjini Bandyopadhyay and Geetha Basappa}

For entangled polymer solutions, the stress relaxation occurs by reptation dynamics with time scale $\tau_{\text {rep }}$, for which

$$
\mu(t)=\sum_{n \text { odd }} \frac{8}{n^{2} \pi^{2}} \mathrm{e}^{\left(\frac{-n^{2} t}{\tau_{\text {rep }}}\right)} .
$$

In a linear creep experiment, a step response is applied ( $\sigma_{x y}=0$ for $t \leq 0$ and $\sigma_{x y}=\sigma_{0}$ for $t>0)$ and strain $\gamma(t)$ is measured, which is a solution of

$$
\sigma_{0}=\int_{0}^{t} G\left(t-t^{\prime}\right) \dot{\gamma}\left(t^{\prime}\right) \mathrm{d} t^{\prime}
$$

The linear response of a viscoelastic material to an applied stress may be determined by a conventional frequency sweep experiment done using a rotating disc or concentric cylinders rheometer. The rheometer applies very small oscillatory stresses and calculates the resultant strain in the sample over the desired frequency range. For an applied angular frequency $\omega$, the response that is in phase with the applied stress is used to calculate the storage modulus $G^{\prime}(\omega)$ while the out of phase component gives the loss modulus $G^{\prime \prime}(\omega)$.

\subsection{Nonlinear rheology}

Nonlinear rheology describes the response of a material to much larger stresses. As the name suggests, the strain induced in a sample varies nonlinearly with the applied stress in this regime. The nonlinear behaviour of a viscoelastic material in steady flow experiments is characterized by shear thinning or thickening, the presence of non-zero yield stress and normal stress differences, flow-induced phase transitions and the phenomenon of shear banding [1-4] as shown schematically in figure 3. In nonlinear step strain experiments, if a large enough step strain $\gamma_{0}$ is applied to a sample, then the stress induced in the sample may be expressed by $\sigma_{x y}(t)=\gamma_{0} G_{n l}\left(t-t^{\prime}, \gamma_{0}\right)$. The normal stresses under these conditions are no longer negligible and must be taken into consideration. For $\gamma_{0} \rightarrow 0, G_{n l} \rightarrow G(t)$ as measured in linear rheology.

Systems of giant wormlike micelles formed in certain surfactant solutions are known to show very unusual nonlinear rheology [1]. In steady shear, the shear stress saturates to a constant value above a critical strain rate $\dot{\gamma}$ (as shown in figure 3d) while the first normal stress difference increases roughly linearly with shear rate [2]. Such behaviour is a signature of mechanical instability of the shear banding type [2,5] and may be understood in terms of the reptation-reaction model which involves the reversible breakage and recombination of wormlike micelles along with repation dynamics known for polymer solutions. Alternatively, the non-monotonicity of the flow curve has been attributed to the coexistence of two thermodynamically stable phases (isotropic and nematic) in the sheared solution [6].

The flow curve may be measured under conditions of controlled stress or strain rate, and depending on the time interval between the collection of data points, we can obtain metastable or steady-state branches, respectively. In stress relaxation experiments, a constant step strain rate is applied to the sample in the nonlinear regime, following which the relaxation of stress in the sample is measured as a function of time. Alternatively, stress relaxation may be studied after cessation of a controlled strain rate that had been applied to the sample for a known duration. 


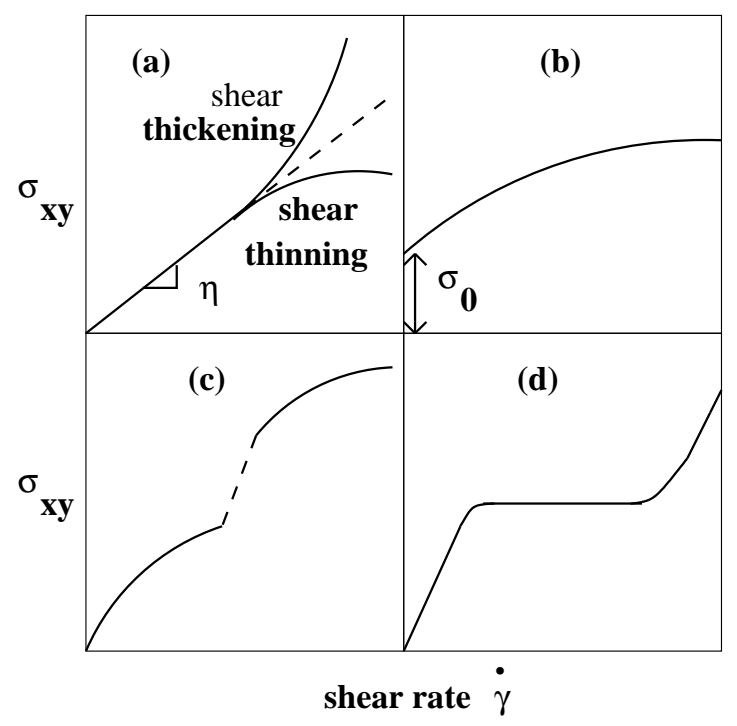

Figure 3. (a) shows Newtonian $\left(\sigma_{x y}=\eta \gamma_{0}\right)$, shear thinning and shear thickening behaviours seen in viscoelastic fluids. (b) shows the flow curve of a Herschel-Bulkley plastic with a finite yield stress $\sigma_{0}$. At $\sigma_{x y}<\sigma_{0}$, the material behaves like a solid. At $\sigma_{x y}>\sigma_{0}, \sigma_{x y}=\sigma_{0}+K_{p} \dot{\gamma}^{p}$, which reduces to the Bingham equation for $p=1$. (c) shows the flow curve of a system undergoing a flow-induced phase transition, characterized by a sudden jump in $\sigma$ as shown by the dotted line. (d) shows a flow curve showing a plateau region, which is a signature of shear banding.

\section{Our experiments on CTAT aqueous solutions}

We have studied the linear and nonlinear rheology of dilute aqueous solutions of the surfactant system CTAT (Cetyltrimethylammonium tosilate) at $25^{\circ} \mathrm{C}$. Above concentrations of $0.04 \mathrm{wt} . \%$, and temperatures of $23^{\circ} \mathrm{C}$ [7], CTAT self-assembles to form cylindrical worm-like micelles which get entangled at concentrations $>0.9 \mathrm{wt} . \%$. The lengths of these wormlike micelles depend on the concentrations of the surfactant and the added salt, the temperature and the energy of scission of the micelle. The energy of scission is the excess free energy of a pair of hemispherical end caps relative to the rod like region containing an equivalent number of surfactant molecules. The number density of the elongated micelles of length $L$ is given by [7,8]

$$
C_{0}(L) \sim \frac{1}{L^{2}} \exp \left(-\frac{L}{L_{\mathrm{avg}}}\right)
$$

where $L$ is expressed in monomer units and

$$
L \sim \phi^{0.5} \exp \left(\frac{E_{\text {scis }}}{2 k_{\mathrm{B}} T}\right),
$$

where $\phi$ is the surfactant volume fraction and $E_{\text {scis }}$ is the energy of scission of the micelle. In these systems, stress relaxation occurs by reptation with time scale $\tau_{\text {rep }}$ (the curvilinear 


\section{A K Sood, Ranjini Bandyopadhyay and Geetha Basappa}

diffusion of the micelle through an imaginary tube segment) as for conventional polymers and by the reversible scission (breakdown and recombination of micelles with time scale $\tau_{b}$ ) [2]. The time scales $\tau_{\text {rep }}$ and $\tau_{b}$ may or may not be comparable and depend on the surfactant concentration, presence of counterions in the solution and temperature.

(a) Macrorheology measurements: The frequency response of a viscoelastic material may be measured using a rheometer which consists of a device that can simultaneously apply a torque and measure the resultant strain. The in-phase and out-of-phase responses of the material to the torque are measured to calculate its elastic and viscous moduli, respectively. The instrument used by us is Rheolyst AR-1000N (T.A. Instruments, U.K.) stress controlled rheometer with temperature control and software for strain rate control to measure the elastic and viscous responses of $1 \mathrm{wt} . \%$ CTAT between the angular frequency range of $0.03 \mathrm{rad} / \mathrm{sec}$ and $10 \mathrm{rad} / \mathrm{sec}$. The rheometer used was equipped with four strain gauge transducers capable of measuring the normal force with an accuracy of $10^{-4} \mathrm{~N}$. The measurements were made using a cone-and-plate geometry of cone diameter $4 \mathrm{~cm}$ and angle $1^{\circ} 59^{\prime \prime}$.

The linear regime of CTAT was first ascertained by looking for a range of stress values where the magnitudes of the response functions were found to be independent of the applied oscillatory stress. The elastic and viscous moduli and the viscosity of CTAT $1 \mathrm{wt} . \%$ at $25^{\circ} \mathrm{C}$ were found to be constant for stresses between 0.05 and $0.1 \mathrm{~Pa}$, oscillating at a frequency of $0.1 \mathrm{~Hz}$. Hence for the linear response measurement, $0.08 \mathrm{~Pa}$ was chosen as the amplitude of the oscillatory stress, oscillating between angular frequencies $0.03 \mathrm{rad} / \mathrm{sec}$ and $10 \mathrm{rad} / \mathrm{sec}$. At frequencies higher than $10 \mathrm{rad} / \mathrm{sec}$, the waveform depicting the strain becomes distorted, possibly due to the slip between the sample and the plates. This, therefore, limits the measurements till $10 \mathrm{rad} / \mathrm{sec}$. Linear response measurements (figure 4) show that at the lowest frequencies, CTAT behaves like a viscous

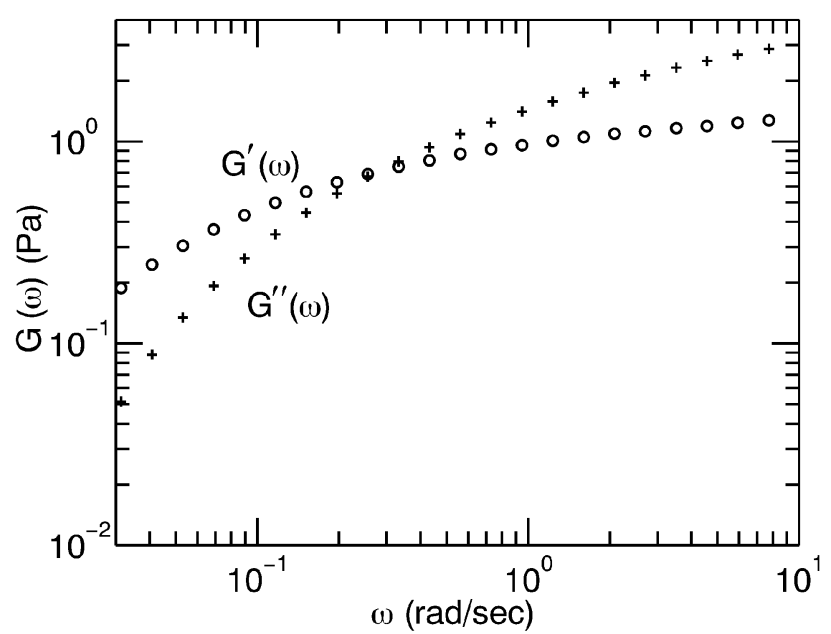

Figure 4. Measurement of the elastic modulus $G^{\prime}(\omega)$ and the viscous modulus $G^{\prime \prime}(\omega)$ of 1 wt.\% CTAT by the method of macrorheology, using a cone and plate rheometer, with applied stress $=0.08 \mathrm{~Pa}$ at $25^{\circ} \mathrm{C}$. 

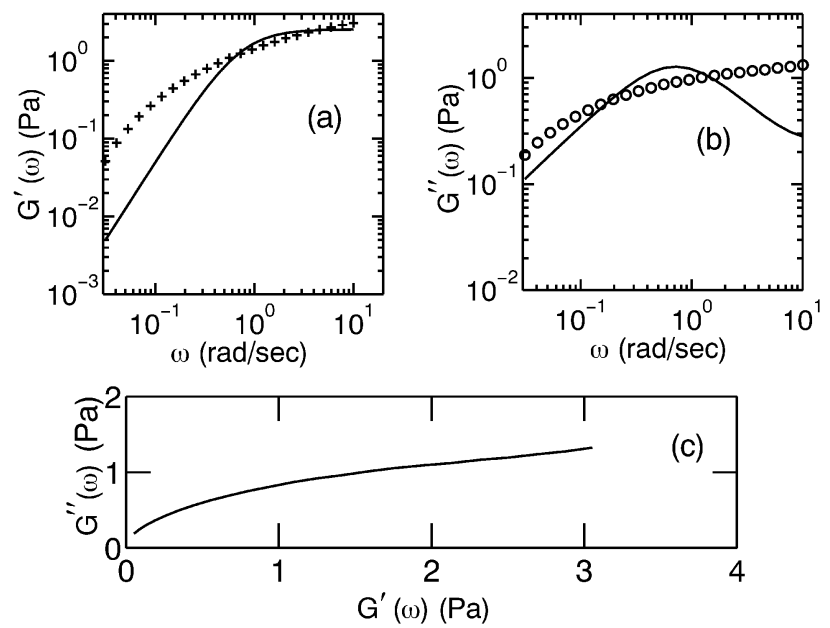

Figure 5. (a) $G^{\prime}(\omega)$ and (b) $G^{\prime \prime}(\omega)$ of 1 wt.\% CTAT using a rheometer (data same as shown in figure 4). The solid lines show the least square fits to the Maxwell model. (c) shows the Cole-Cole plot which deviates from the semicircle exhibited by Maxwellian systems.

material, whereas in higher frequency runs, the behaviour is found to be predominantly elastic. The crossover is found to occur at $0.45 \mathrm{rad} / \mathrm{sec}$ which corresponds to a relaxation time $\tau_{R}$ of 2.2 seconds. Cates et al [8] have shown that for a system of wormlike micelles, like $\mathrm{CPyCl} / \mathrm{NaCl}, G^{\prime}(\omega)$ and $G^{\prime \prime}(\omega)$ are given by Maxwell model:

$$
\begin{aligned}
G^{\prime}(\omega) & =G_{0} \omega^{2} \tau_{R}^{2} /\left(1+\omega^{2} \tau_{R}^{2}\right), \\
G^{\prime \prime}(\omega) & =G_{0} \omega \tau_{R} /\left(1+\omega^{2} \tau_{R}^{2}\right),
\end{aligned}
$$

where $\tau_{R}=\left(\tau_{b} \tau_{\text {rep }}\right)^{1 / 2}$. Figure $5 \mathrm{a}$ and $\mathrm{b}$ show the least square fits of the data to the Maxwell model giving $G_{0}=2.1 \mathrm{~Pa}$ and $\tau_{R}=2.2 \mathrm{sec}$. We find that for CTAT at concentration $1 \mathrm{wt} . \%$, the fit is very poor. Further, the Cole-Cole plot (figure 5c) corresponding to the above data shows a deviation from the semi-circular behaviour expected in Maxwellian systems and shows an upturn at high frequencies. This deviation from Maxwellian behaviour is possibly due to the comparable values of $\tau_{\text {rep }}$ and $\tau_{b}$ in this system unlike in other wormlike micellar systems where the differences in the time scales $\left(\tau_{b} \ll \tau_{\text {rep }}\right)$, lead to a 'motional averaging' effect. We have tried the Doi-Edwards model where [9]

$$
\begin{aligned}
G^{\prime}(\omega) & =G_{0} \sum_{p \text { odd }}\left(\omega \tau_{D} / p\right)^{2} /\left(1+\left(\omega \tau_{D} / p\right)^{2}\right), \\
G^{\prime \prime}(\omega) & =G_{0} \sum_{p \text { odd }}\left(\omega \tau_{D} / p\right) /\left(1+\left(\omega \tau_{D} / p\right)^{2}\right) .
\end{aligned}
$$

Here also, the fit with $p=1$ and 3 is poor as shown in figure 6 . The Doi-Edwards model gives $G_{0} \sim 3 \mathrm{~Pa}$ and $\tau_{D} \sim 1 \mathrm{sec}$. We also find that the Hess model which is given by [9]

$$
\begin{aligned}
G^{\prime}(\omega) & =\left(\left(\eta_{0}-\eta_{\infty}\right) / \tau_{\epsilon}\right) \omega^{2} \tau_{\epsilon}^{2} /\left(1+\omega^{2} \tau_{\epsilon}^{2}\right), \\
G^{\prime \prime}(\omega) & =\left(\left(\eta_{0}-\eta_{\infty}\right) / \tau_{\epsilon}\right) \omega \tau_{\epsilon} /\left(1+\omega^{2} \tau_{\epsilon}^{2}\right)
\end{aligned}
$$




\section{A K Sood, Ranjini Bandyopadhyay and Geetha Basappa}
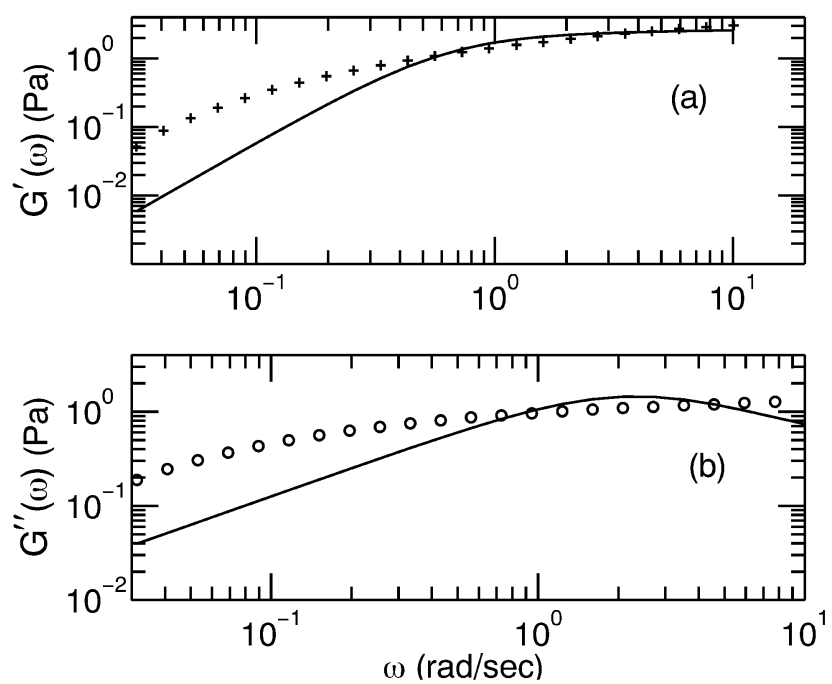

Figure 6. Data same as in figure 4 and the corresponding fits to the Doi-Edwards model shown by solid lines.

does not fit our data over the entire frequency range. In this model, $\eta_{\infty}$ is the high frequency shear viscosity and $\tau_{\epsilon}$ is a characteristic relaxation time of the system. It is likely that $G^{\prime}(\omega)$ and $G^{\prime \prime}(\omega)$ can be fitted with a model calculated in low and high frequency regions separately, as is usually done in polymer literature [10]. Linear response measurements were also made for CTAT $1.9 \mathrm{wt} . \%$ and CTAT $5 \mathrm{wt} . \%$. Interestingly, CTAT $1.9 \mathrm{wt} . \%$ shows an anomalously large relaxation time, whereas CTAT $5 \mathrm{wt} . \%$ is found to exhibit Maxwellian behaviour, as also seen in recent studies [9].

(b) Microrheology measurements: In recent years, micro-rheological techniques have been developed in addition to macroscopic rheometry measurements using rotating disc or concentric cylinder rheometer. The basic idea behind microrheology is the tracking or manipulation of sub-micrometer particles immersed in the viscoelastic medium to be studied. Using magnetic beads as the probe particles, which can be manipulated by magnetic field gradients, the viscoelastic properties of $F$-actin networks [11] and the vitreous body of the eye [12] have been measured. It is also possible to do microrheological measurements by a quantitative measurement of the mean square displacement $\left\langle\Delta r^{2}(t)\right\rangle$ of the probe particles caused due to thermal fluctuations. This can be done either using laser interferometry with a resolution less than $1 \mathrm{~nm}[13,14]$ or by diffusing wave spectroscopy [15].

The motion of the probe particle of radius $a$ may be described by a generalized Langevin equation given by

$$
m \dot{v}(t)=f_{R}(t)-\int_{0}^{\infty} \zeta(t-\tau) v(\tau) \mathrm{d} \tau,
$$

where $m \dot{v}(t)$ is the inertia of the particle, $f_{R}(t)$ is the contribution due to electrostatic and Brownian forces on the particle. $\zeta$ defines a time-dependent memory function which contributes to the viscous damping of the particle in the viscoelastic medium. The memory 


\section{Rheology of wormlike micelles}

function $\zeta(t)$ and $f_{R}(t)$ are related by the following temporal autocorrelation function:

$$
\left\langle f_{R}(0) f_{R}(t)\right\rangle=k_{\mathrm{B}} T \zeta(t),
$$

where $k_{\mathrm{B}}$ is the Boltzmann's constant and $T$ is the temperature.

In the frequency domain, the viscosity of the medium may be related to the frequency dependent memory function by the generalized Stokes-Einstein relation

$$
\widetilde{\eta}(s)=\frac{\widetilde{\zeta}(s)}{6 \pi a},
$$

where $s$ is the complex frequency given by $s=i \omega$. The complex viscoelastic modulus is given by [15]

$$
\widetilde{G}(s)=s \widetilde{\eta}(s)=\frac{s}{6 \pi a}\left[\frac{6 k_{\mathrm{B}} T}{s^{2}\left\langle\Delta r^{2}(s)\right\rangle}-m s\right] .
$$

The last term on the right hand side of eq. (11) is the contribution due to the inertia of the particle and can be omitted except at very high frequencies. $\left\langle\Delta r^{2}(t)\right\rangle$ of the probe particle is obtained from the intensity autocorrelation function which can be measured in diffusing wave spectroscopy experiments in the transmission or backscattering geometries. The Laplace transform of $\left\langle\Delta r^{2}(t)\right\rangle$ is used to calculate $\widetilde{G}(s)$ using eq. (11). $\widetilde{G}(s)$ is then fitted to a functional form in $s$, which may then be used to calculate $G^{\star}(\omega)$, using the method of analytic continuation.

We have used microrheology to estimate the $G^{\prime}(\omega)$ and $G^{\prime \prime}(\omega)$ of an aqueous solution of CTAT of weight fraction $1 \%$. The probe particles used are polystyrene colloidal particles of diameter $0.23 \mu \mathrm{m}$ dispersed in water at $\phi=1 \%$. Diffusing wave spectroscopy was performed on the equilibrated sample using our light scattering setup consisting of a $\mathrm{Kr}^{+}$ ion laser (model 2020, Spectra Physics, U.S.A., excitation wavelength used $647.1 \mathrm{~nm}$ ), a homemade spectrometer, photomultiplier tube (model R943-02, Hamamatsu, Japan), single photon amplifier discriminator (SPEX) and a MALVERN 7132 CE 64 channel correlator (figure 7). The light scattered $I_{s}(t)$ by the probe particles in the backscattering direction at a temperature of $25^{\circ} \mathrm{C}$ is used to measure the normalized intensity autocorrelation function $g_{2}(t)=\left\langle I_{s}(0) I_{s}(t)\right\rangle /\left\langle I_{s}(0)\right\rangle^{2}$, as shown in figure 8a. For backscattering geometry, $g_{2}(t) \sim \mathrm{e}^{-\Gamma\left(k^{2}\left\langle\Delta r^{2}(t)\right\rangle\right)^{1 / 2}}$ [16], which is used to get $\left\langle\Delta r^{2}(t)\right\rangle$ as shown in figure 8b,

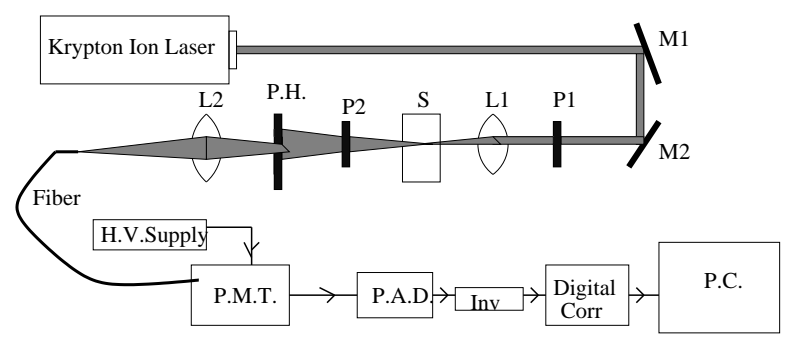

Figure 7. Our light scattering setup in the transmission geometry: P1 and P2 are the analyser and polariser respectively, L1 and L2 are convex lenses of $f=20$ and $30 \mathrm{~mm}$ respectively, P.H. is a pin hole, $\mathrm{S}$ the sample that scatters light and Inv is an inverter circuit. M1 and M2 are plane mirrors that steer the incident laser beam. 

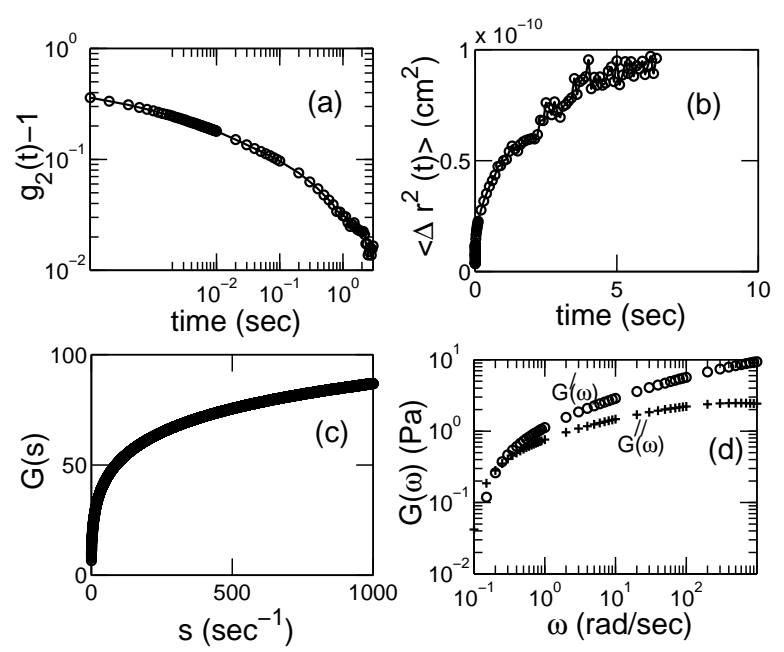

Figure 8. The microrheology results: (a) shows the correlation function in the backscattering direction, (b) the $\left\langle\Delta r^{2}(t)\right\rangle$, (c) the complex modulus $G(s)$ and (d) shows the calculated values of $G^{\prime}(\omega)$ and $G^{\prime \prime}(\omega)$.

using $\Gamma=2$. The parameter $\Gamma$ is a constant depending on the polarization of the scattered light and varies inversely with the transport mean free path $l^{\star}$ of the diffusing photon. Figure $8 \mathrm{c}$ shows $G(s)$ calculated using eq. (11), which was fitted to $G(s)=p_{0}+p_{1} s^{-0.55}+$ $p_{2} s^{0.3}+p_{3} s^{0.5}+p_{4} s$ [15]. Putting $s=i \omega, G^{\prime}(\omega)$ and $G^{\prime \prime}(\omega)$ of the dispersing gel are calculated as shown in figure $8 \mathrm{~d}$. Comparison of figures 4 and $8 \mathrm{~d}$ shows similar magnitudes of the viscoelastic response functions obtained by macrorheology and microrheology methods. Further, in figure 8d, the crossover of the viscous and elastic moduli occur at $\omega_{\mathrm{co}} \sim 0.4 \mathrm{rad} / \mathrm{sec}$, indicating a relaxation time $\tau_{R} \sim 2.5$ seconds for $1 \%$ CTAT at $25^{\circ} \mathrm{C}$, similar to macrorheology measurements. It may be noted that microrheology may be used to calculate the frequency response of CTAT to much higher frequencies than the conventional rheometer experiment.

\section{Nonlinear rheology of CTAT}

To study the nonlinear rheology of CTAT, we have measured the flow curve of CTAT 1.35 wt. $\%$ at $25^{\circ} \mathrm{C}$ as shown in figure 9 . The measurements are done under conditions of controlled stress. The data points are collected at intervals of 1 second, a value comparable to the relaxation time of the sample. The resultant branch of the measured flow curve is metastable, and its existence was demonstrated by Grand et al [5]. The flow curve is found to saturate to a constant stress value above a critical shear rate $\dot{\gamma}_{c}$, while the first normal stress difference is found to increase linearly with shear rate. The plateau of the shear stress at high shear rates in $\mathrm{CPyCl} / \mathrm{NaSal}$ has been interpreted by Grand et al [5] as a characteristic feature of the flow curves of complex fluids that gives rise to a mechanical instability of the nature of shear banding [2]. Shear banding results in the formation of bands of high and low viscosities in the sample, supporting low and high 


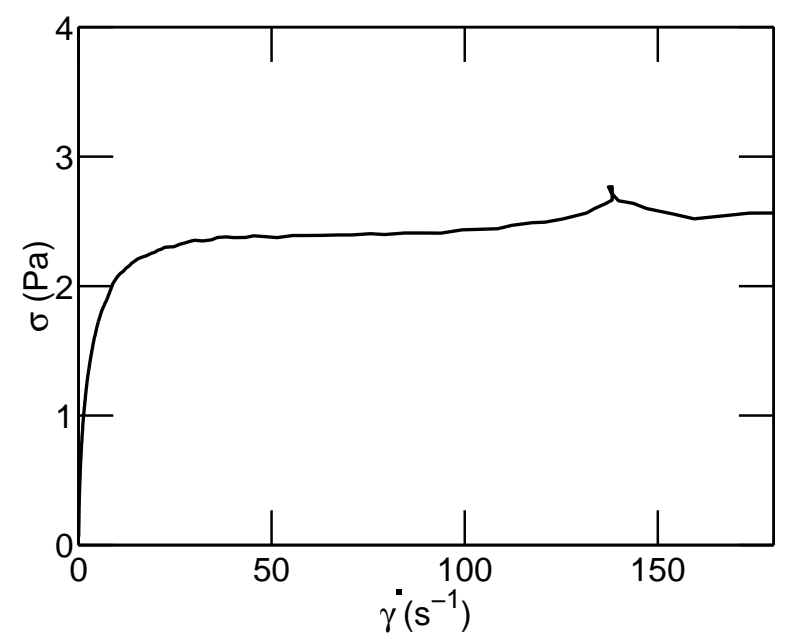

Figure 9. The metastable branch of the flow curve of $1.35 \mathrm{wt} . \%$ CTAT, measured under conditions of controlled stress.

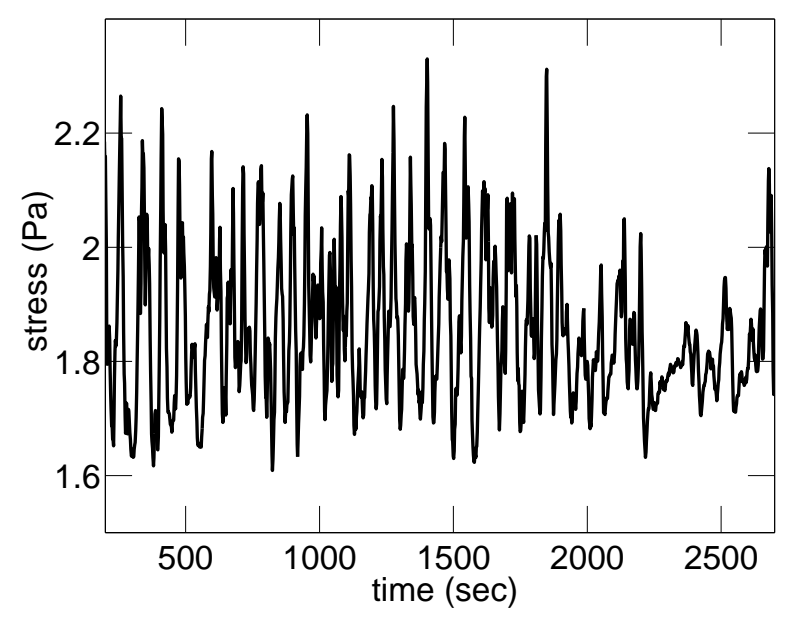

Figure 10. The time-dependent relaxation of stress in $1.35 \mathrm{wt} . \%$ CTAT, on subjecting the sample to a constant step-strain rate of $100 \mathrm{~s}^{-1}$.

shear rates, respectively. However, the same phenomenon observed in CTAB $/ \mathrm{NaSal}$ at a higher concentration has been explained by Berret et al [3] as due to the coexistence of isotropic and nematic phases in the sheared sample.

In addition to the measurement of the flow curve for our system, we have studied the stress relaxation in the sample after subjecting it to a step strain rate. At $25^{\circ} \mathrm{C}$, on applying controlled shear rates whose values lie in the plateau region of the flow curve, the stress, instead of decaying to a steady state, is found to oscillate in time. Figure 10 shows the time dependent stress relaxation in the $1.35 \mathrm{wt} . \%$ CTAT sample at $25^{\circ} \mathrm{C}$, on subjecting the sample to a step strain rate of $100 \mathrm{~s}^{-1}$. The Fourier spectra of these time-dependent signals 


\section{A K Sood, Ranjini Bandyopadhyay and Geetha Basappa}

show time scales of the order of a few tens of seconds, which are an order of magnitude larger than $\tau_{R}$.

We identify the observed time-dependent behaviour as a manifestation of the mechanical instability due to the formation of shear bands [2,5]. Preliminary analysis of the time series obtained from the stress relaxation experiments done in the nonlinear regime shows the existence of positive Lyapunov exponents [17] and finite correlation dimensions [18] ( $>2$ at shear rates $>75 \mathrm{~s}^{-1}$ ), which points to the existence of deterministic chaos in sheared aqueous solutions of CTAT. The Lyapunov exponent characterizes the divergence of stress trajectories in the system, whereas the correlation dimension gives us information about the geometry of the attractor on which the trajectories in phase space asymptotically lie. On increasing the temperature of the sample to $35^{\circ} \mathrm{C}$, and on maintaining the same shear rates as in the previous experiments done at $25^{\circ} \mathrm{C}$, the time dependent oscillations in the stress relaxation are found to disappear completely. This is in accordance with previous studies on the temperature dependence of the flow curve of $\mathrm{CPyCl} / \mathrm{NaSal}$ [3] which shows a decrease in the width of the plateau with increasing temperature. The disappearance of the time-dependent behaviour in sheared CTAT at higher temperatures is thus a direct consequence of the disappearance of the shear bands in the sample [3]. We have done extensive studies on the time-dependence of the stress relaxation of dilute, aqueous, sheared solutions of CTAT by doing more elaborate analysis of the time-series obtained from our experiments [19].

\section{Acknowledgements}

AKS thanks the Board of Research in Nuclear Sciences and RB thanks the CSIR for financial support. The authors thank Sriram Ramaswamy, P R Nott and V Kumaran for the use of the rheometer. They would like to acknowledge M E Cates' lectures on rheology delivered at the Summer School on Soft Condensed Matter, International Center of Theoretical Physics, Italy, May 4-June 5, 1998.

\section{References}

[1] H Rehage and H Hoffmann, Mol. Phys. 74, 933 (1991)

[2] N A Spenley, M E Cates and T C B McLeish, Phys. Rev. Lett. 71, 939 (1993)

[3] L F Berret, D C Roux and G Porte, J. Phys. II 4, 1261 (1994)

[4] P Fischer and H Rehage, Rheol. Acta 36, 13 (1997)

[5] G Grand, J Arrault and M E Cates, J. Phys. II France 7, 1071 (1997)

[6] L F Berret, Langmuir 13, 2227 (1997)

[7] J F A Soltero, J E Puig, O Manero and P C Schulz, Langmuir 11, 3337 (1995)

[8] M E Cates and S J Candau, J. Phys. Condens. Matter 2, 6869 (1990)

[9] J F A Soltero, J E Puig and O Manero, Langmuir 12, 2654 (1996)

[10] M Doi and S F Edwards in The Theory of Polymer Dynamics (Oxford University Press, New York, 1986)

[11] K S Zaner and P A Valberg, J. Cell Biol. 109, 2233 (1989)

[12] B Lee, M Litt and G Buchsbaum, Biorheology 29, 521 (1993)

[13] F Gittes, B Scnurr, P D Olmsted, F C Mackintosh and C F Schmidt, Phys. Rev. Lett. 79, 3286 (1997)

[14] B Schnurr, F Gittes, F C Mackintosh and C F Schmidt, Macromol. 30, 7781 (1997)

[15] T G Mason and D Weitz, Phys. Rev. Lett. 74, 1250 (1995) 


\section{Rheology of wormlike micelles}

[16] D J Pine, D A Weitz, G Maret, P E Wolf, E Herbolzheimer and P M Chaikin in Scattering and Localization of Classical Waves in Random Media edited by Ping Sheng (World Scientific, USA, 1990)

[17] J Gao and Z Zheng, Phys. Rev. E 49, 3807 (1994)

[18] P Grassberger and I Procaccia, Phys. Rev. Lett. 50, 346 (1983)

[19] Ranjini Bandyopadhyay, Geetha Basappa and A K Sood (to be published) 